\title{
Structure of metal-rich (001) surfaces of III-V compound semiconductors
}

\author{
C. Kumpf, ${ }^{*}$ D. Smilgies, ${ }^{\dagger}$ E. Landemark,${ }^{\ddagger}$ M. Nielsen, and R. Feidenhans’1 \\ Materials Research Department, Risb National Laboratory, DK-4000 Roskilde, Denmark \\ O. Bunk, J. H. Zeysing, Y. Su, and R. L. Johnson \\ II. Institut für Experimentalphysik, Universität Hamburg, D-22761 Hamburg, Germany \\ L. Cao and J. Zegenhagen \\ European Synchrotron Radiation Facility, Boîte Postale 220, F-38043 Grenoble Cedex, France \\ B. O. Fimland \\ Department of Physical Electronics, Norwegian University of Science and Technology, N-7491 Trondheim, Norway \\ L. D. Marks \\ Department of Material Science and Engineering, Northwestern University, Evanston, Illinois 60208 \\ D. Ellis \\ Department of Chemistry, Northwestern University, Evanston, Illinois 60208
}

(Received 22 February 2001; published 17 July 2001)

\begin{abstract}
The atomic structure of the group-III-rich surface of III-V semiconductor compounds has been under intense debate for many years, yet none of the models agrees with the experimental data available. Here we present a model for the three-dimensional structure of the $(001)-c(8 \times 2)$ reconstruction on InSb, InAs, and GaAs surfaces based on surface x-ray diffraction data that was analyzed by direct methods and subsequent least squares refinement. Contrary to common belief the main building blocks of the structure are not dimers on the surface but subsurface dimers in the second bilayer. This essential feature of the structure is accompanied by linear arrays of atoms on nonbulklike sites at the surface which, depending on the compounds, exhibit a certain degree of disorder. A tendency to group-III-dimer formation within these chains increases when descending the periodic table. We propose that all the $c(8 \times 2)$ reconstructions of III-V semiconductor surfaces contain the same essential building blocks.
\end{abstract}

DOI: 10.1103/PhysRevB.64.075307

PACS number(s): 68.35.Bs, 61.10.-i, 81.05.-t

\section{INTRODUCTION}

Within the last decade III-V compound semiconductors have become extremely relevant for fabricating high-speed electronic and optoelectronic devices. Layer growth and epitaxial behavior, which are strongly influenced by the morphology of the substrate surface, play an important role for the fabrication process. Thus, detailed knowledge about the geometric structure of the surface is essential. Depending on preparation, temperature, and especially whether group-IIIor group-V-rich conditions are dominant, the (001) surfaces of III-V compound semiconductors show a wealth of different surface reconstructions. For the group-V-rich surfaces, group- $\mathrm{V}$ dimers have been clearly identified as the basic building blocks in these reconstructions. McConville and coworkers saw $\mathrm{Sb}-\mathrm{Sb}$ dimers on the $\operatorname{InSb}(001)-c(4 \times 4)$ surface in scanning tunneling microscopy (STM) images ${ }^{1}$ whereas Göthelid et al. found As dimers in the $\operatorname{InAs}(001)-\beta 2-(2 \times 4)$ structure using surface $\mathrm{X}$-ray diffraction (SXRD). ${ }^{2}$ These surface structures were strongly supported recently by density-functional theory and firstprinciples electronic structure calculations. ${ }^{3,4}$ It has also been generally believed that the group-III-rich surface consists of (group-III) dimers, ${ }^{5}$ but no agreement on a specific model exists in the literature. In an early publication, based on high- resolution photoelectron spectroscopy (PES), John and coworkers proposed an InSb- $\beta-c(8 \times 2)$ model consisting of six In-In dimers, three within each $(4 \times 2)$ subcell. $^{6}$ Figure 1(a) illustrates this so-called missing-dimer model. This model was supported for InSb and GaAs by various experimental methods, most frequently STM and low-energy electron diffraction (LEED). ${ }^{1,7}$ However, it was also questioned, e.g., by Varekamp et al. in a STM study on InSb. ${ }^{8}$ Biegelsen et al. modified the model by suggesting only two dimers in the top layer and one in the second bilayer ${ }^{9}$ [see Fig. 1(b)]. This so-called $\beta 2$ model was proposed on the basis of a STM study on the GaAs surface, and was supported later by both experimental ${ }^{10}$ and theoretical studies. ${ }^{11}$ Another variant, the $\beta 3$ model for InAs [see Fig. 1(c)], was suggested by Ohkouchi and Ikoma. ${ }^{12}$ It contains one top-layer and two second-bilayer dimers and was favored by a combined LEED and STM study. ${ }^{13}$ All these models have a common feature: Group-III surface dimers are the primary structural element. However, the features in the STM images interpreted as dimers have never been uniquely identified as really being made up of two atoms, as in the case of group-Vrich surfaces. Furthermore, most of the STM measurements suggested a $(4 \times 1)$ or $(4 \times 2)$ unit cell $1^{1,10,12,14}$ whereas all diffraction experiments clearly showed a $c(8 \times 2)$ structure. This was attributed to defects ${ }^{13}$ and often a coexistence of 
(a) $\beta-c(8 \times 2)$, John et al.

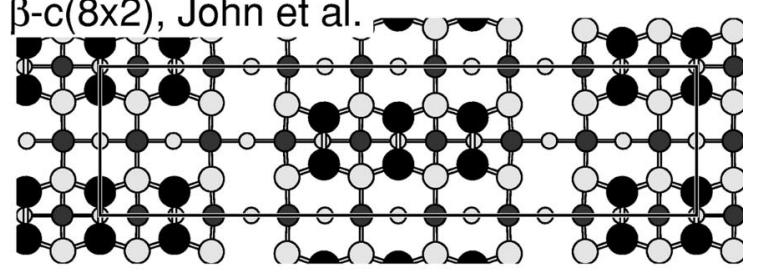

(b) $\beta 2-c(8 \times 2)$, Biegelsen et al.

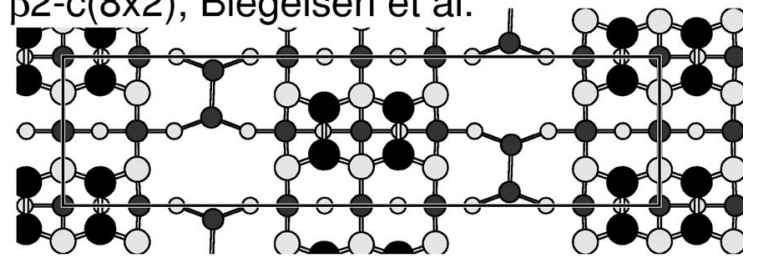

(c) $\beta 3-c(8 \times 2)$, Ohkouchi et al.

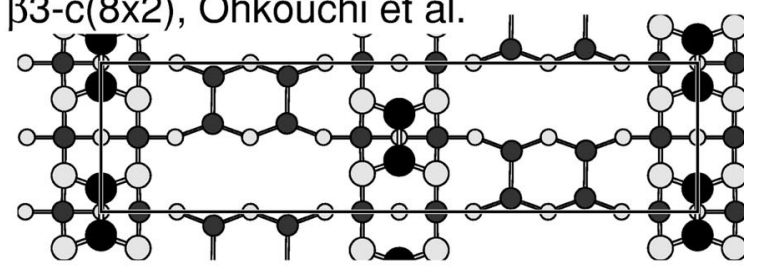

(d) Skala et al.

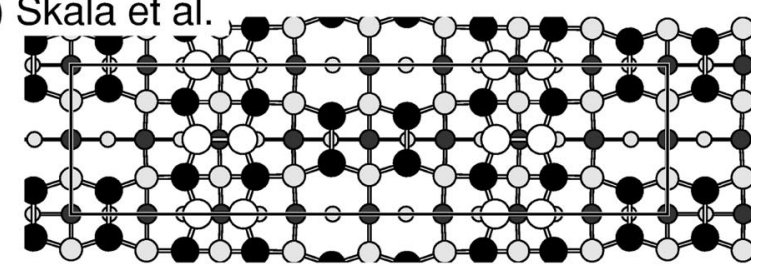

(e) Jones et al.

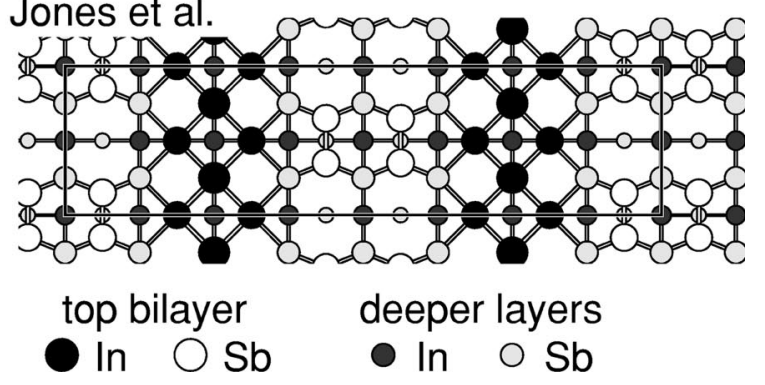

FIG. 1. Popular models from the literature for the $c(8 \times 2)$ reconstructed In-rich (001) surface of InSb: (a) $\beta$ model (missingdimer model) of John et al. (Ref. 6) (1989), (b) $\beta 2$ model of Biegelsen et al. (Ref. 9) (1990), (c) $\beta 3$ model of Ohkouchi and Ikoma (Ref. 12) (1994), (d) model of Skala et al. (Ref. 16) (1993), and (e) model of Jones et al. (Ref. 17) (1998).

$(4 \times 2)$ and $c(8 \times 2)$ reconstructed regions was assumed. ${ }^{1,8,13}$ In more recent STM work on InSb additional weak features indicating the $c(8 \times 2)$ unit cell were found. ${ }^{8,15}$

Skala and co-workers modified the group-III-dimer model suggesting additionally group- $\mathrm{V}$ dimers on top of double rows of group-III atoms, ${ }^{16}$ a model again based on STM measurements on GaAs [see Fig. 1(d)]. Five years later this model was seized again in a SXRD study on InSb by Jones and co-workers. ${ }^{17}$ They replaced the group- $\mathrm{V}$ dimers by single group-III atoms forming chains along the [110] axis which are separated from each other by group-V dimers [see Fig. 1(e)]. Their model contains no group-III dimers and was supported by a reinterpretation of STM images. ${ }^{15}$ Recently a completely different model was proposed independently by two groups; theoretical total-energy calculations combined with a LEED analysis ${ }^{18}$ by one group and direct methods applied to SXRD data by the other. ${ }^{19}$ The present article is an extension of the latter work. Characteristic features of this model are subsurface dimerization of group-III atoms in the second bilayer and linear arrangements of atoms at the surface. The subsurface dimerization is the only attribute producing a $c(8 \times 2)$ periodicity and explains the contradiction between the $(4 \times 2)$ unit cell observed in STM and the $c(8$ $\times 2$ ) diffraction patterns. Group-III dimers in the top layer are present on GaAs but not on the heavier III-V compounds In $\mathrm{As}$ and InSb.

A fundamental problem with structure determination is to identify the correct solution; when refining a structure it is rarely clear whether a local or the global minimum of the goodness-of-fit function has been found. For bulk structure determination using $\mathrm{x}$-ray diffraction data, this ambiguity has been largely removed using what are called direct methods. ${ }^{20}$ Direct methods find probable values for the phases of the measured reflections, consistent with the fact that the scattering arises from atoms. Combining these phases with the measured amplitudes allows approximate maps of the charge density to be calculated. Placing atoms at the peaks in these maps gives a first approximation to the structure, and will almost always yield a fairly good fit to the data. In many cases not all atoms appear at first, but by using Fourier difference methods the rest of the structure can be found without much difficulty. The power of direct methods is that they eliminate the need to guess a model for the structure; they yield a set of plausible structures against which subsequent refinements are carried out. While direct methods have been used successfully on two-dimensional (in-plane) SXRD data or transmission electron surface diffraction data, ${ }^{21}$ extending them to three-dimensional SXRD data is not simple. One reason for this is that large out-of-plane data sets are necessary for finding stable solutions with a three-dimensional direct methods code. But since it is often too time consuming - even at modern synchrotron-light sources- to obtain a full-size out-of-plane data set it is common to measure a fairly complete two-dimensional data set (typically $80 \%$ of the available reflections) and only a rather limited number of fractional-order rods. The small number of threedimensional measurements makes it harder to obtain stable solutions. We have recently shown that the mathematical approach of "feasible sets" 22 developed for image restoration problems can be applied to crystallographic problems, ${ }^{23}$ permitting additional constraints to be introduced that enable full, $a b$ initio, surface structure determination to be performed in three dimensions. ${ }^{24}$ Since this is a truly modelindependent approach, group-III dimers will only arise in the data analysis if they are an intrinsic part of the structure.

\section{EXPERIMENT}

Several data sets on (001) surfaces of three types of III-V semiconductor, GaAs, InAs, and InSb, were measured. InSb and InAs samples were prepared in an ultrahigh vacuum 


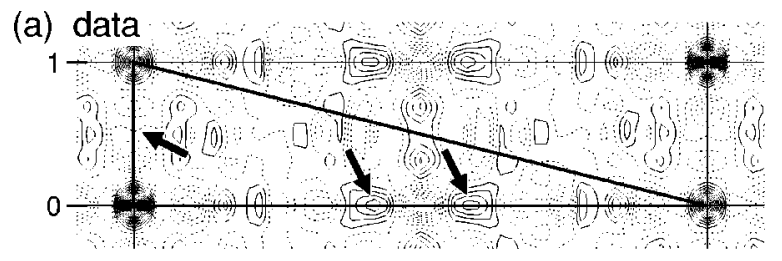

(b) our model

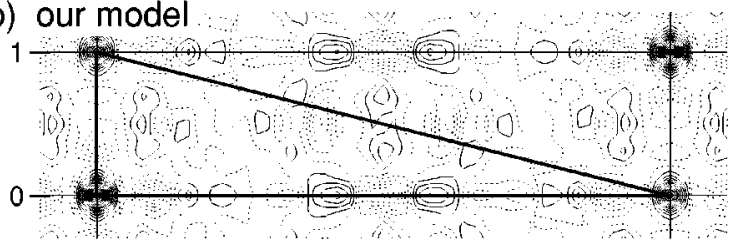

(c) Biegelsen et al.

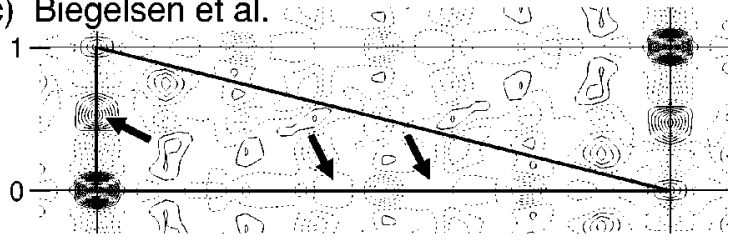

(d) Skala et al.

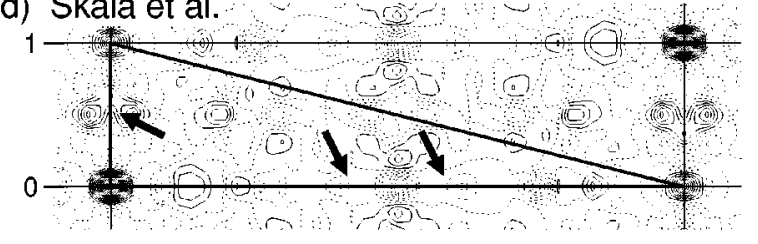

(e) Jones et al.

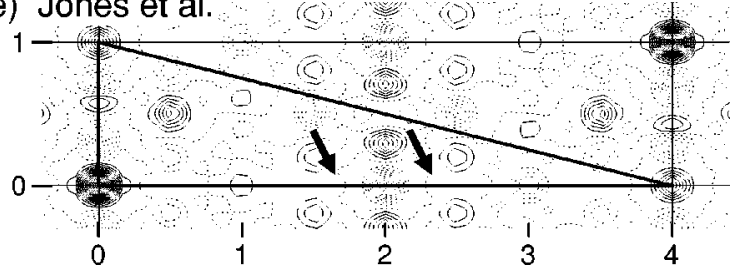

FIG. 2. Experimental Patterson map calculated from our measured in-plane data set (a) and theoretical Patterson maps calculated from our model (b) and from different models from literature: (c) $\beta 2$ model of Biegelsen et al., (Ref. 9), (d) model of Skala et al. (Ref. 16), and (e) model of Jones et al. (Ref. 17).

(UHV) system using the standard surface preparation technique of repeated cycles of argon-ion bombardment and annealing at the appropriate temperature. GaAs surfaces were prepared by using $\operatorname{GaAs}(001)$ wafers, the surfaces of which had been grown by molecular beam epitaxy (MBE) and which had been covered by a protective layer of amorphous arsenic immediately after the growth. Before the SXRD measurements, the cap layer was desorbed in UHV at $450^{\circ} \mathrm{C}$. Subsequent annealing for $5 \mathrm{~min}$ at $490^{\circ} \mathrm{C}$ yielded a $\operatorname{GaAs}(001)-c(8 \times 2)$ reconstruction as confirmed by LEED. Scanning tunneling microscopy was used to ensure that the GaAs(001) surfaces used for the SXRD measurements were well ordered and uniformly $c(8 \times 2)$ reconstructed. Figure 9 below shows a representative STM image from the GaAs(001) surface. More STM images from MBE-grown GaAs(001) surfaces prepared by thermal desorption of an As capping layer are presented in Ref. 25 and they are similar to the images from samples prepared using other techniques. ${ }^{6,14}$ After preparation the samples were transferred to a small portable UHV chamber which was mounted on the $z$-axis
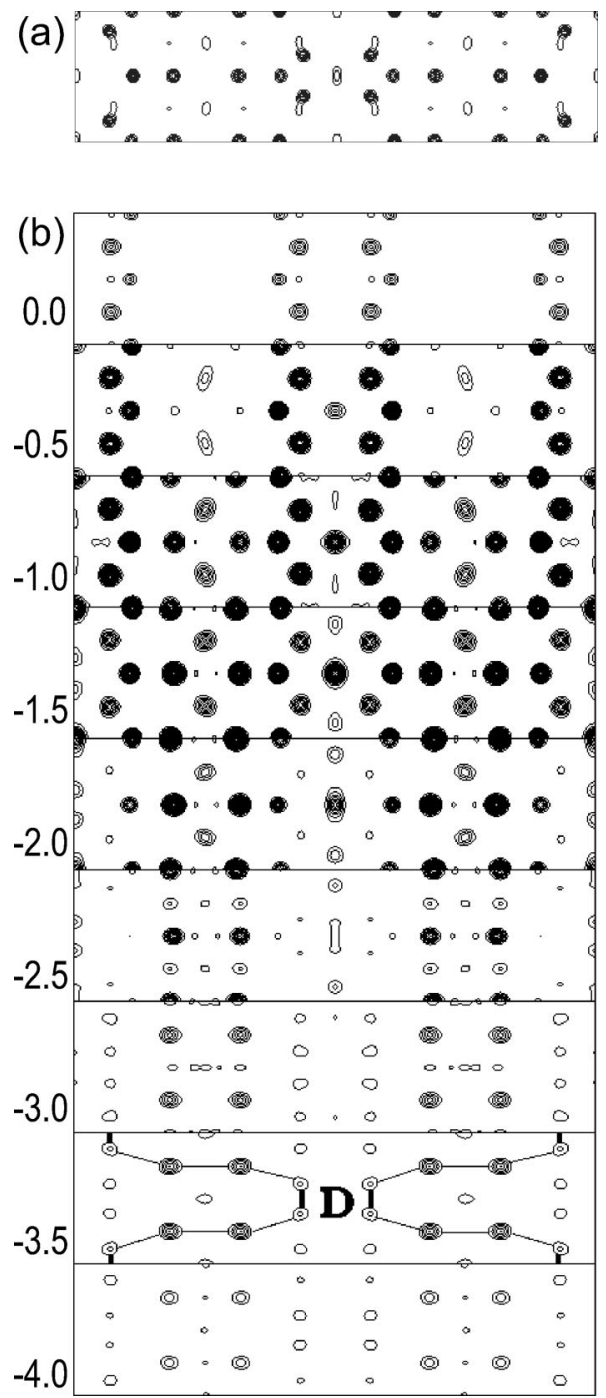

FIG. 3. (a) Contour map of the charge density distribution in the $x-y$ plane obtained from the two-dimensional direct method code, using in-plane data only. Each maximum corresponds to the position of an atom in the uppermost layer. One $c(8 \times 2)$ unit cell is shown with the origin located at the lower left. (b) Two-dimensional sections of the three-dimensional map showing contours of the charge density in $x-y$ planes at different heights $z$. Numbers in the left margin indicate the $z$ coordinate in $\AA ; z=0$ corresponds to the height of the uppermost In atoms on an unreconstructed Interminated surface. In the second bilayer the dimers are marked $D$ and lines between the atoms are drawn to guide the eye.

diffractometer on the BW2 wiggler beamline at the Hamburger Synchrotronstrahlungslabor (HASYLAB) and aligned with respect to the incident $\mathrm{x}$-ray beam. The glancing angle was $0.6^{\circ}, 0.5^{\circ}$, and $0.2^{\circ}$ for the measurements on InSb, GaAs, and InAs, respectively. On all samples extended inplane and out-of-plane data sets were measured at wavelengths between $1.24 \AA$ and $1.42 \AA$. The intensity of each reflection was determined by rotating the sample about its surface normal ( $\omega$ scans). The peaks were integrated, background subtracted, and corrected in the standard manner for Lorentz and polarization factors, active sample area, and rod intercept. ${ }^{26}$ The (001) surface of the zinc-blende structure has 

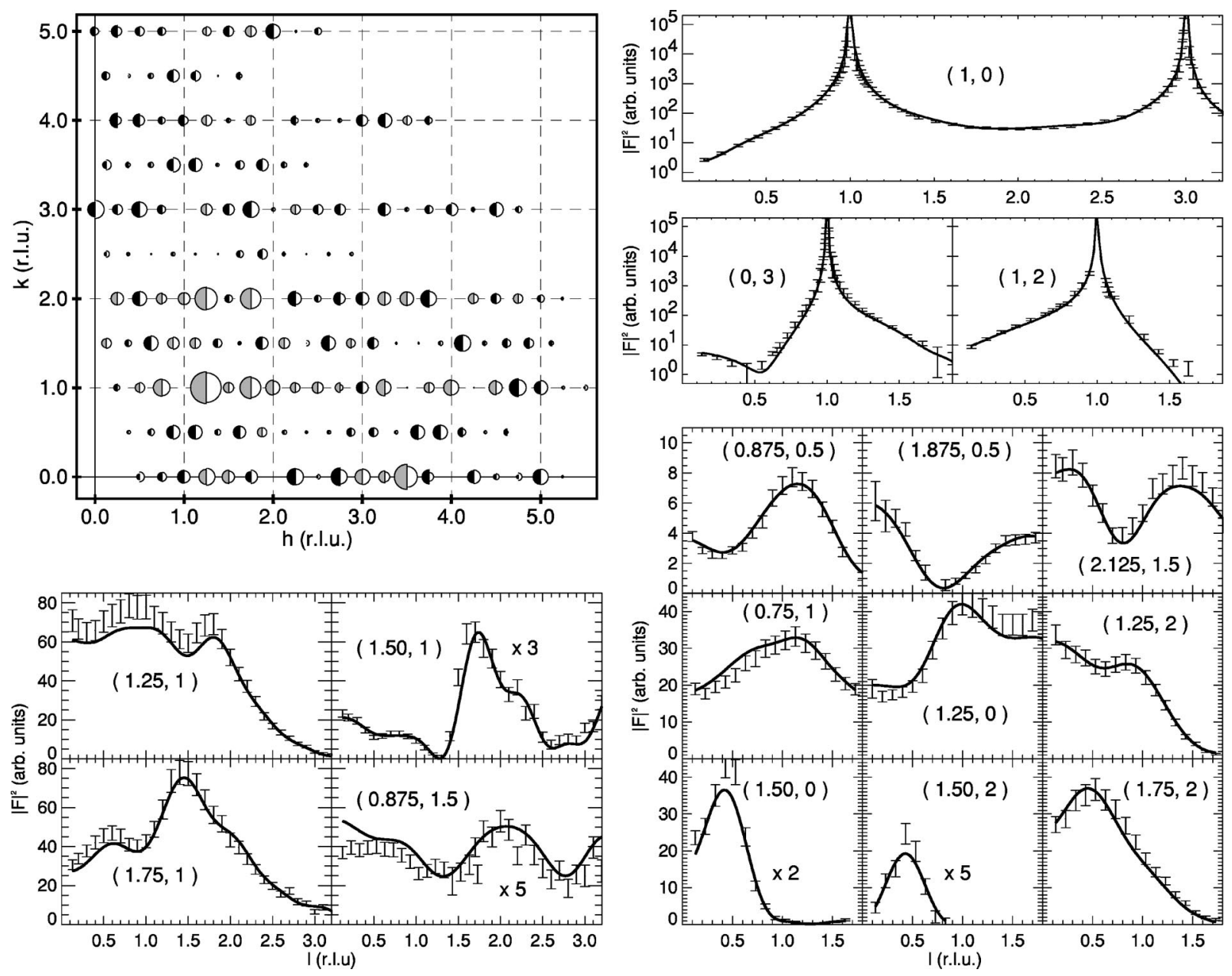

FIG. 4. InSb data set. Upper left: Plot of the in-plane data. The areas of the filled and open semicircles represent measured and calculated intensities, respectively. Gray/white circles have been scaled by a factor of 0.5. Upper right: integer-order rods. Bottom: fractional-order rods.

only twofold rotational symmetry; thus there is only one rotational domain. By averaging equivalent reflections using a $c 2 \mathrm{~mm}$ symmetry systematic errors in $|F|^{2}$ of $\epsilon=7.8 \%$, $7.2 \%$, and $10.2 \%$ were determined for the in-plane data sets of InSb, GaAs, and InAs, respectively. The final data sets consisted of 171, 112, and 75 nonequivalent in-plane reflections, 14, 17, and 18 fractional-order rods with 282, 548, and 566 reflections as well as three, two, and five crystal truncation rods (CTR's) with 137, 71, and 375 reflections (InSb, GaAs, and InAs, respectively). On the InSb data set a (00l) rod was measured as well (95 reflections) but not included in the refined data set for reasons discussed below. The eighthorder reflections from InAs were so broad that their integrated intensities could not be evaluated, so the InAs inplane data set consisted only of reflections from the $(4 \times 1)$ subcell. Standard LEED coordinates $\left(\mathbf{a}=(1 / 2)[1 \overline{1} 0]_{\text {bulk }}, \mathbf{b}\right.$ $\left.=(1 / 2)[110]_{\text {bulk }}, \mathbf{c}=[001]_{\text {bulk }}\right)$ are used in the following.

\section{DATA ANALYSIS AND RESULTS}

\section{A. Patterson function}

For solving surface structures considerable insight can be gained from a contour plot of the Patterson function

$$
P(x, y) \propto \sum_{h, k}\left|F_{h k 0}\right|^{2} \cos [2 \pi(h x+k y)]
$$

where $\left|F_{h k 0}\right|^{2}$ is the measured intensity of the in-plane reflections $(h k 0)$. The "experimental Patterson map" calculated from the InSb data is shown in Fig. 2(a). Each positive peak in the map corresponds to an interatomic distance vector in the unit cell projected onto the surface plane. Several clearly separated peaks can be seen in the irreducible unit. As a first test for the correctness of a specific model we can compare the experimental Patterson map of Fig. 2(a) with a "theoretical Patterson map" calculated on the basis of the model. Equation (1) gives the theoretical Patterson function if the measured structure factors are replaced by those calculated from the model, using the same subset of $(h k 0)$ reflections. In Fig. 2(b) a contour plot of such a theoretical Patterson function is shown for the model presented in this work. Comparison with (a) reveals excellent agreement between the experimental and theoretical Patterson maps. Figures 2(c-e) are theoretical Patterson maps for the most popular models from the literature. The arrows on the left axis of the figure mark a peak at $(0,0.55)$ arising from the dimer distance in the Biegelsen and Skala models. This peak is also 


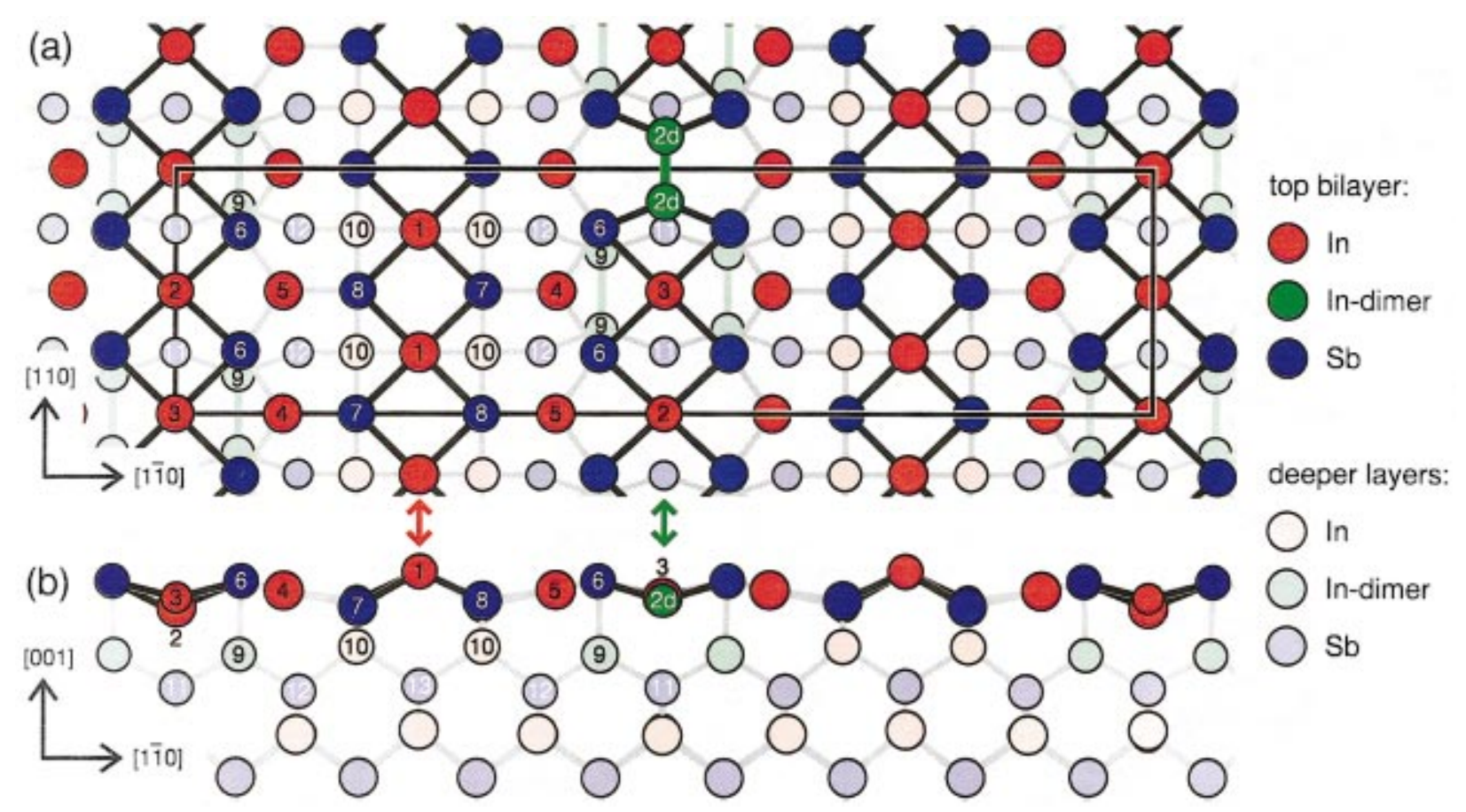

FIG. 5. (Color) Structural model for InSb viewed from above (a) and from the side (b). In and Sb atoms are shown as red and blue circles. In dimers are colored green. Atoms in deeper layers are indicated by pale colors. The main atoms are labeled and the dimer site In $2 d$ is shown at only one location. All gray bars represent covalent In-Sb bonds; other bonds are indicated schematically.

present in the theoretical Patterson maps of the models by John et al. and Ohkouchi and Ikoma (not shown). The absence of the peak in the experimental Patterson function is clear evidence that surface dimers in the $y$ direction (i.e., group-III dimers) are not present in this reconstruction. Other significant differences between experimental and theoretical Patterson maps occur at $(1.7,0)$ and $(2.3,0)$, where relatively strong peaks in the experimental Patterson plot are not reproduced in the theoretical plots [Figs. 2(c-e)]. Some other features are reflected quite well by the Jones model, e.g., the peaks at approx $(2,0.25),(2,0.75)$, and $(0.5,0.5)$, even though they are weaker in the experimental Patterson plot. In summary, judging from the Patterson maps, the Jones model has some correct features, but other models containing group-III dimers as the basic building block can be eliminated.

\section{B. Direct methods}

To find a starting model for the refinement of our data we did not try to modify models from the literature but used direct methods. We first analyzed the InSb surface, a reasonably complete $c(8 \times 2)$ data set, using both the two- and the three-dimensional direct method code. We found a solution to the structure relatively straightforwardly in two dimensions [Fig. 3(a)] and in three [Fig. 3(b)]. In both cases the solution was unambiguous and placing atoms at the peaks in the maps gave relatively low $R$ factors confirming that the data set used is of high quality. Contrary to the models from literature described above we found an indium dimer only in the second bilayer, not in the top layer. The upper layer contains rows of In atoms along $\mathbf{b}$ and, with only a small occupancy, an indium dimer (site In $2 d$, see Fig. 5 below) within these rows. Since the two types of atom in InSb (as well as in $\mathrm{GaAs}$ ) have almost the same number of electrons it was still unclear at this point in the data analysis which type of atom occupies which site. But the InAs data allowed this question to be answered, as will be discussed below. The second fairly complete data set is that of GaAs. The result of twodimensional (2D) direct methods showed the same basic elements as for InSb except that the sites corresponding to In1 and In 2 were absent and the In $2 d$ dimer site had a higher occupancy. The three-dimensional solutions were just about stable, indicating the same structure we found for InSb, but with different occupancies. Because of missing eighth-order reflections the last data set, InAs, did not yield a solution in three-dimensional direct methods, although the twodimensional results were similar to those for GaAs and InSb. In conclusion, the direct methods yielded one common model for InSb and GaAs, which was used as a starting model for refinement with a least-squares algorithm.

\section{Refinement}

We first discuss the result of the refinements for the InSb data set. Refining the positions of the uppermost three InSb bilayers and the occupancy for the sites In 1 and In $2 d$ (those showing a reduced occupancy in direct methods) results in $\chi^{2}=3.95$ for the whole data set and indicates that the model found in direct methods is basically correct. This first fit was close to the starting model, i.e., to the direct methods result, and could not be improved significantly by refining the occupancies of other atoms within the top bilayer. Note that the starting model based on direct methods was derived from fractional-order data only. However, the model was immedi- 
TABLE I. Atomic parameters for the $(001)-c(8 \times 2)$ reconstruction of InSb, GaAs, and InAs. Positions are given in LEED coordinates, Debye Waller (DW) factors in $\AA^{2}$. All DW factors are isotropic, except for atoms at site 1 in the InAs structure, which shows a large in-plane disorder. DW factors not listed in the table were fixed at bulk values: $\mathrm{DW}(\mathrm{In})=1.60 \AA^{2}, \mathrm{DW}(\mathrm{Ga})=1.43 \AA^{2}, \mathrm{DW}(\mathrm{Sb})=1.25 \AA^{2}$, and $\mathrm{DW}(\mathrm{As})=0.87 \AA^{2}$. Standard deviations are calculated assuming uncorrelated parameters. Values listed without errors were not refined due to $c 2 \mathrm{~mm}$ symmetry constraints.

\begin{tabular}{|c|c|c|c|}
\hline Site & InSb: position; DW $\left(\AA^{2}\right)$ & GaAs: position; DW $\left(\AA^{2}\right)$ & InAs: position; DW $\left(\AA^{2}\right)$ \\
\hline $1 \mathrm{In} / \mathrm{Ga}$ & $2.000,0.500,-0.051(4) ; 8.6(5)$ & $2.000,0.500,0.077(4) ; 1.8(4)$ & $2.000,0.500,0.004(1){ }^{\mathrm{a}}$ \\
\hline $2 \mathrm{In} / \mathrm{Ga}$ & $0.000,1.000,-0.295(4) ; 7.3(4)$ & not occupied & $0.000,1.000,-0.251(1) ; 3.14(13)$ \\
\hline $2 d \mathrm{In} / \mathrm{Ga}$ & $0.000,0.730(6),-0.208(7) ; 7.2(8)$ & $0.000,0.707(2),-0.275(1) ; 2.8(2)$ & not occupied \\
\hline $3 \mathrm{In} / \mathrm{Ga}$ & $0.000,0.000,-0.203(3) ; 6.4(3)$ & not occupied & $0.000,0.000,-0.101(1) ; 6.57(17)$ \\
\hline $4 \mathrm{In} / \mathrm{Ga}$ & $0.881(1), 0.000,-0.159(2) ; 1.58(10)$ & $0.870(1), 0.000,-0.171(1) ; 2.60(9)$ & $0.902(1), 0.000,-0.166(1) ; 5.46(11)$ \\
\hline $5 \mathrm{In} / \mathrm{Ga}$ & $0.884(1), 1.000,-0.162(2) ; 1.58(10)$ & $0.883(1), 1.000,-0.179(1) ; 1.54(8)$ & $0.923(1), 1.000,-0.108(1) ; 0.23(6)$ \\
\hline $6 \mathrm{Sb} / \mathrm{As}$ & $0.532(1), 0.511(1),-0.106(1) ; 1.77(8)$ & $0.540(1), 0.511(1),-0.111(1) ; 1.18(4)$ & $0.563(1), 0.482(3),-0.089(1) ; 2.67(6)$ \\
\hline $7 \mathrm{Sb} / \mathrm{As}$ & $1.485(1), 0.000,-0.220(2) ; 1.49(11)$ & $1.461(1), 0.000,-0.241(1) ; 0.58(6)$ & $1.529(1), 0.000,-0.238(1) ; 2.32(15)$ \\
\hline $8 \mathrm{Sb} / \mathrm{As}$ & $1.493(1), 1.000,-0.255(2) ; 1.67(12)$ & $1.474(1), 1.000,-0.235(1) ; 1.45(8)$ & $1.474(1), 1.000,-0.251(1) ; 1.93(12)$ \\
\hline $9 \mathrm{In} / \mathrm{Ga}$ & $0.516(1), 0.315(1),-0.536(2) ; 2.33(7)$ & $0.526(1), 0.330(1),-0.538(1) ; 2.03(6)$ & $0.519(0), 0.334(1),-0.511(0) ; 1.98(5)$ \\
\hline $10 \mathrm{In} / \mathrm{Ga}$ & $1.483(1), 0.500(1),-0.490(1) ; 1.76(7)$ & $1.484(1), 0.530(1),-0.494(1) ; 1.14(5)$ & $1.498(0), 0.489(3),-0.502(0) ; 1.76(5)$ \\
\hline $11 \mathrm{Sb} / \mathrm{As}$ & $0.000,0.495(1),-0.730(1) ; 0.79(8)$ & $0.000,0.492(1),-0.742(1) ; 0.37(5)$ & $0.000,0.500(4),-0.733(1) ; 1.23(8)$ \\
\hline $12 \mathrm{Sb} / \mathrm{As}$ & $1.008(1), 0.494(1),-0.750(1) ; 1.29(6)$ & $1.013(0), 0.498(1),-0.769(0) ; 0.45(4)$ & $1.001(0), 0.500(4),-0.757(1) ; 1.50(7)$ \\
\hline $13 \mathrm{Sb} / \mathrm{As}$ & $2.000,0.500,-0.720(1) ; 1.02(8)$ & $2.000,0.500,-0.730(1) ; 0.37(5)$ & $2.000,0.500,-0.751(1) ; 1.76(8)$ \\
\hline $\mathrm{In} / \mathrm{Ga}$ & $0.000,0.000,-1.007(2)$ & $0.000,0.000,-0.992(1)$ & $0.000,0.000,-0.970(1)$ \\
\hline $\mathrm{In} / \mathrm{Ga}$ & $1.026(1), 0.000,-0.999(1)$ & $1.017(1), 0.000,-1.025(1)$ & $1.002(1), 0.000,-1.011(1)$ \\
\hline $\mathrm{In} / \mathrm{Ga}$ & $1.996(1), 1.000,-0.975(1)$ & $1.994(1), 1.000,-0.982(1)$ & $1.995(2), 1.000,-1.000(0)$ \\
\hline $\mathrm{In} / \mathrm{Ga}$ & $0.987(1), 1.000,-1.002(2)$ & $1.000(1), 1.000,-0.999(1)$ & $1.012(1), 1.000,-0.991(1)$ \\
\hline $\mathrm{In} / \mathrm{Ga}$ & $0.000,1.000,-0.972(2)$ & $0.000,1.000,-1.011(1)$ & $0.000,1.000,-0.992(1)$ \\
\hline $\mathrm{Sb} / \mathrm{As}$ & $0.508(1), 0.000,-1.253(1)$ & $3.505(1), 0.000,-1.256(1)$ & $0.515(1), 0.000,-1.248(1)$ \\
\hline $\mathrm{Sb} / \mathrm{As}$ & $1.526(1), 0.000,-1.251(2)$ & $1.524(1), 0.000,-1.252(1)$ & $1.503(1), 0.000,-1.234(1)$ \\
\hline $\mathrm{Sb} / \mathrm{As}$ & $1.502(1), 1.000,-1.249(2)$ & $1.509(1), 1.000,-1.253(1)$ & $1.505(1), 1.000,-1.270(1)$ \\
\hline $\mathrm{Sb} / \mathrm{As}$ & $0.491(1), 1.000,-1.246(1)$ & $0.495(1), 1.000,-1.249(1)$ & $0.474(1), 1.000,-1.245(1)$ \\
\hline $\mathrm{In} / \mathrm{Ga}$ & $0.498(1), 0.494(1),-1.500(1)$ & $0.497(1), 0.511(1),-1.502(1)$ & $0.495(0), 0.516(2),-1.493(0)$ \\
\hline $\mathrm{In} / \mathrm{Ga}$ & $1.509(1), 0.501(1),-1.498(1)$ & $1.509(1), 0.480(1),-1.500(1)$ & $1.503(0), 0.465(1),-1.502(0)$ \\
\hline $\mathrm{Sb} / \mathrm{As}$ & $2.000,0.500,-1.748(1)$ & $2.000,0.500,-1.756(1)$ & $2.000,0.500,-1.754(1)$ \\
\hline $\mathrm{Sb} / \mathrm{As}$ & $1.003(1), 0.503(1),-1.742(1)$ & $1.001(0), 0.506(1),-1.747(0)$ & $0.997(0), 0.482(5),-1.747(1)$ \\
\hline $\mathrm{Sb} / \mathrm{As}$ & $0.000,0.499(1),-1.744(1)$ & $0.000,0.496(1),-1.753(1)$ & $0.000,0.500(4),-1.750(1)$ \\
\hline $\mathrm{In} / \mathrm{Ga}$ & $1.996(1), 0.000,-2.003(1)$ & $1.996(1), 0.000,-2.003(1)$ & $2.000(2), 0.000,-2.004(0)$ \\
\hline $\mathrm{In} / \mathrm{Ga}$ & $1.000(1), 1.000,-1.997(2)$ & $1.001(1), 1.000,-1.998(1)$ & $0.994(1), 1.000,-2.004(1)$ \\
\hline $\mathrm{In} / \mathrm{Ga}$ & $1.001(1), 0.000,-1.990(1)$ & $1.001(1), 0.000,-1.995(1)$ & $1.000(1), 0.000,-1.991(1)$ \\
\hline $\mathrm{In} / \mathrm{Ga}$ & $0.000,1.000,-1.993(2)$ & $0.000,1.000,-1.999(1)$ & $0.000,1.000,-1.998(1)$ \\
\hline $\mathrm{In} / \mathrm{Ga}$ & $0.000,0.000,-2.004(2)$ & $0.000,0.000,-2.002(1)$ & $0.000,0.000,-1.998(1)$ \\
\hline $\mathrm{Sb} / \mathrm{As}$ & $1.500(1), 1.000,-2.250(2)$ & $1.500(1), 1.000,-2.250(1)$ & $1.500(1), 1.000,-2.250(1)$ \\
\hline $\mathrm{Sb} / \mathrm{As}$ & $1.500(1), 0.000,-2.250(2)$ & $1.500(1), 0.000,-2.250(1)$ & $1.500(1), 0.000,-2.250(1)$ \\
\hline $\mathrm{Sb} / \mathrm{As}$ & $0.500(1), 1.000,-2.250(1)$ & $0.500(1), 1.000,-2.250(1)$ & $0.500(1), 1.000,-2.250(1)$ \\
\hline $\mathrm{Sb} / \mathrm{As}$ & $0.500(1), 0.000,-2.250(1)$ & $0.500(1), 0.000,-2.250(1)$ & $0.500(1), 0.000,-2.250(1)$ \\
\hline
\end{tabular}

${ }^{\mathrm{a}}$ This site has anisotropic DW factors: $\mathrm{DW}_{x}=4.3(1) \AA^{2}, \mathrm{DW}_{y}=200 \AA^{2}$ (not refined), and $\mathrm{DW}_{z}=5.5(3) \AA^{2}$.

ately able to fit the CTR's as well, which provides additional evidence for its correctness. The best fit depicted in Fig. 4 with $\chi^{2}=2.40$ was achieved by including the atomic positions of two more bilayers in the refinement and allowing the (isotropic) Debye-Waller (DW) factors for all atoms in the two uppermost bilayers to be adjusted. The topmost layers of the corresponding structural model are shown in Fig. 5. Deeper layers showed only very small deviations from bulk positions. The atomic parameters are listed in Table I. In Fig. 6 we present the experimental data for the $\mathrm{InSb}(00 l)$ rod and a calculation based on our model (solid line). If we in- clude this rod in our data set $\chi^{2}$ increases to 3.49 ; further refinement of the model brings $\chi^{2}$ down to 3.15 which is still significantly worse than the value of 2.40 obtained for the data set without the $(00 l)$ rod. It should be noted that in general the $(00 l)$ rod is hard to fit, since everything on the surface including unreconstructed areas and inhomogeneities contributes to it. Hence we left out the $(00 l)$ rod in the quantitative analysis of the surface structure.

The refinement of the GaAs data set was as straightforward as for InSb and the same basic model was employed, but with different DW factors and occupancy of some atomic 


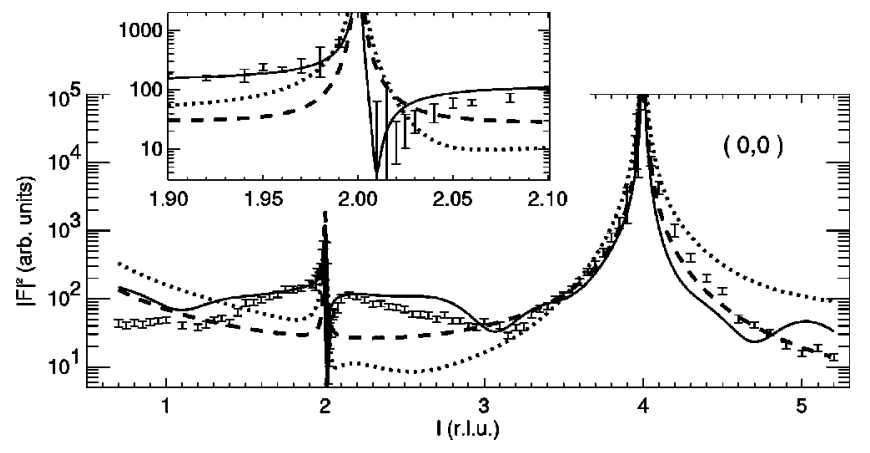

FIG. 6. $(00 l)$ rod from the $c(8 \times 2) \operatorname{InSb}(001)$ surface (data points with error bars) and the curve calculated with our model (solid line). Note the good agreement around the weak (002) reflection shown in the inset. For comparison, calculations for a truncated InSb crystal (dashed line) and the missing-dimer model (Ref. 6) (dotted line) are shown.

sites. Several tests were carried out to evaluate the occupancies for all top-layer atoms. As a result the sites In2 and In3 are not occupied in this structure and the site In1 has a reduced occupancy of $19 \%$. However, unlike in the InSb structure, the dimer site In $2 d$ is occupied to $63 \%$ (see Table II). The first fit-positions of three layers and occupancies of In1 and In $2 d$ were refined-yielded $\chi^{2}=3.32$ and could be improved to $\chi^{2}=2.18$ by including more bulk layers and DW factors. Fractional- and integer-order rods for this fit are shown as solid lines in Fig. 7. The model is only slightly different from the one proposed on the basis of theoretical considerations by Lee et al. ${ }^{18}$ The main differences are the occupancies of sites $\operatorname{In} 1$ and $\operatorname{In} 2 d$, which are fixed to $0 \%$ and $100 \%$ in their model (see also Table II). The dotted lines in Fig. 7 correspond to a fit with this constraint. Only small differences can be seen between the two fits in the figure; however, the difference in the goodness of fit is significant $\left(\chi^{2}=3.14\right.$ when fitting the Lee model to our data).

Refining the InAs data was more problematic because of ambiguity in the position of the In 1 site due, in part, to the missing eighth-order reflections. No good fit could be achieved by refining only three bilayers Even a fit with the set of parameters that gave the "best fits" for InSb and GaAs-positions of five bilayers, isotropic DW factors for two bilayers, and occupancies for the critical sites In1-3-

TABLE II. Comparison of the occupancies of the sites labeled In $1, \operatorname{In} 2, \operatorname{In} 2 d$, and In 3 for the three different surfaces. For InAs and GaAs the results for two different fits are listed, with the corresponding $\chi^{2}$ values. Standard deviations are calculated assuming uncorrelated parameters. Values listed without errors were not refined.

\begin{tabular}{lccccc}
\hline \hline & In1 & In2 & In2d & In3 & $\chi^{2}$ \\
\hline InSb & $57(1) \%$ & $72(2) \%$ & $28(1) \%$ & $100 \%$ & 2.40 \\
InAs & $100 \%$ & $100 \%$ & $0 \%$ & $100 \%$ & 2.71 \\
InAs & $100 \%$ & $68(7) \%$ & $13(4) \%$ & $100 \%$ & 2.55 \\
GaAs & $19(1) \%$ & $0 \%$ & $63(1) \%$ & $0 \%$ & 2.18 \\
GaAs (Ref. 18) & $0 \%$ & $0 \%$ & $100 \%$ & $0 \%$ & 3.14 \\
\hline \hline
\end{tabular}

resulted in $\chi^{2}=11.2$. Satisfactory fits could only be achieved by introducing anisotropic DW factors for site In1 with a large $y$ component. This indicates disorder along the $[110]_{\text {bulk }}$ direction at this site and explains the diffuse eighthorder reflections. As a consequence it was difficult to find exact values for the occupancies of the uppermost sites. The best fit we could achieve, with $70 \%$ occupancy for In 2 and $13 \%$ for $\operatorname{In} 2 d$, yielded $\chi^{2}=2.55$, but fixing these sites at $100 \%$ and $0 \%$, respectively, did not increase $\chi^{2}$ significantly $\left(\chi^{2}=2.71\right.$; see Table II $)$.

Since In and Sb have nearly the same number of electrons they scatter x-rays almost equally strongly, as do Ga and As. From x-ray diffraction results on InSb or GaAs it is therefore difficult to judge which site is occupied by group-III atoms and which by group V. But with the InAs data set it was possible to determine the identity of the atoms. From prior knowledge about the orientation of the $c(8 \times 2)$ cell relative to the bulk the atom type was clear for all sites except for the uppermost ones (In1-5, Sb6-8). Despite the ambiguous results from the InAs data set concerning the In1 site, the fit to the InAs data set was sufficient to identify the atoms of all sites with 100\% occupancy, i.e., all sites except In1-3. The determination was performed by replacing all top-layer atoms (In and As) in the final InAs model with $\mathrm{Nb}$ (which is located halfway between In and As in the periodic table) and calculating the density difference map for this model-i.e., the difference between the 2D electron density of the model and the 2D electron density resulting from the measurement, neglecting differences in the phases:

$$
\begin{aligned}
\triangle \rho(x, y, 0)= & \rho_{\text {expt }}(x, y, 0)-\rho_{\text {model }}(x, y, 0) \\
\propto & \sum_{h, k}\left(\left|F_{h k 0, \text { expt }}\right|-\left|F_{h k 0, \text { model }}\right|\right) \\
& \times \cos \left[2 \pi(h x+k y)-\varphi_{h k 0}\right],
\end{aligned}
$$

where $\varphi_{h k 0}$ is the phase of the structure factor $F_{h k 0}$ $=\left|F_{h k 0}\right| \exp \left(i \varphi_{h k 0}\right)$, calculated from the model. A contour plot of the differences is shown in Fig. 8. Each In (As) atom appears as a positive (negative) peak because its electron density is higher (lower) than that of $\mathrm{Nb}$. It can clearly be seen that In atoms are located at site In4 $(0.9,0)$ and In5 $(0.9,1)$ and As at As1 $(0.5,0.5)$, As2 $(0,1.6)$, and As3 (0,2.4). The atoms on sites In1-3 cannot be identified from this plot due to their reduced occupancy. But from the bond lengths, STM images, and the fact that the surface is In rich it is most likely that they are indium.

\section{DISCUSSION}

A unique model for the group-III-rich (001) surface of III-V compound semiconductors has been found, based on measurements on three different compounds InSb, GaAs, and InAs. The model is in excellent agreement with recent LEED measurements and density-functional theory. ${ }^{18}$ It is significantly different from all previous models in a number of features, most prominent of which is the subsurface dimerization in the second bilayer (site In9). The dimer distances (2.89 $\AA$ for In-In in InSb, $2.64 \AA$ for Ga-Ga in 


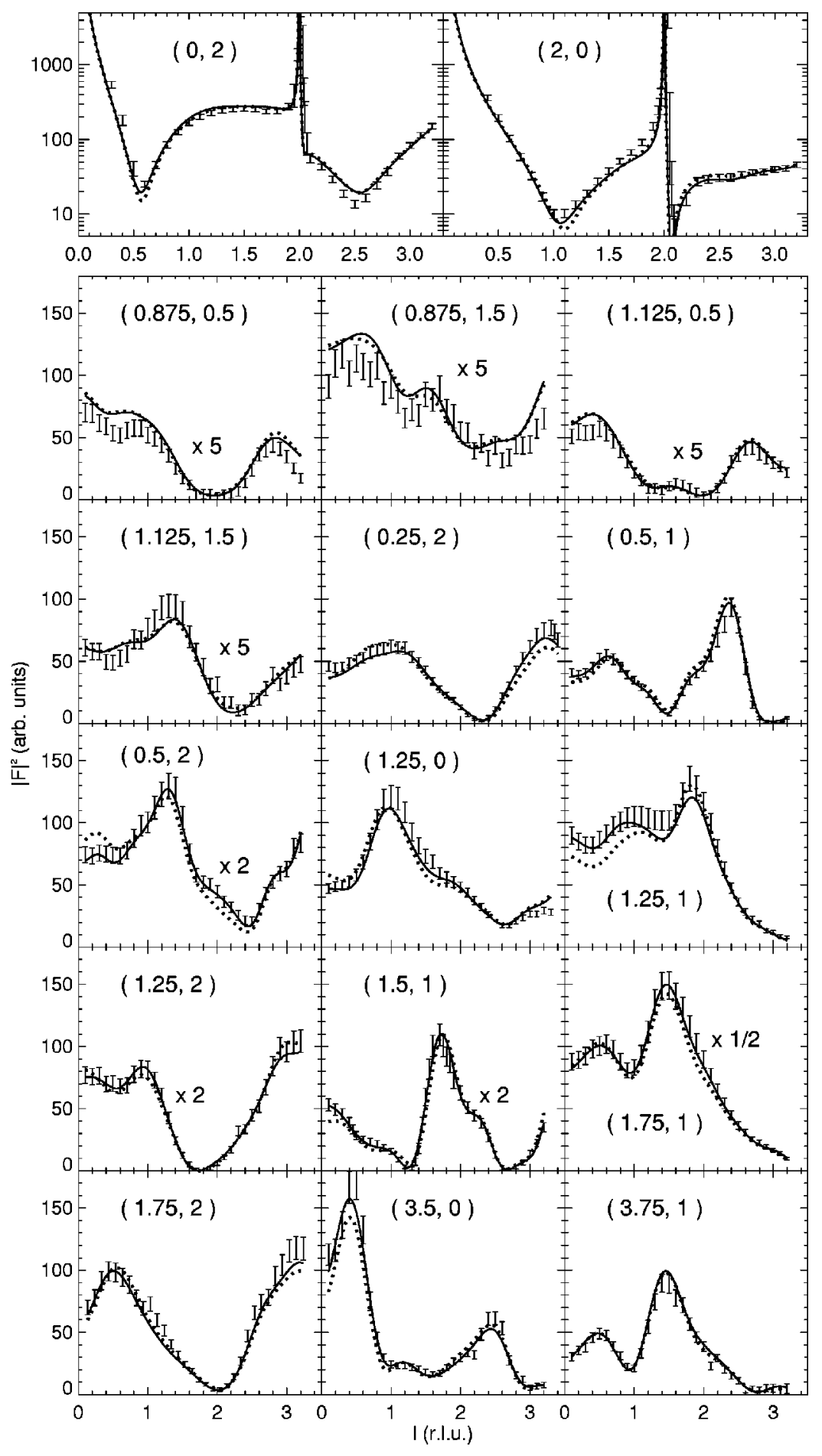

FIG. 7. Integer- and fractional-order rods from the GaAs data set: The error bars represent measured intensities, the solid line is calculated using our best fit. The dotted line is calculated using a model (Ref. 18) that differs from ours in the occupancy of the sites $1(0 \%)$ and $2 d$ $(100 \%)$ (see Table II).

GaAs) are in very good agreement with covalent bond lengths from literature (2.88 $\AA$ for In, $2.54 \AA$ for Ga). The ordering of these subsurface dimers is the only distinct element with a $c(8 \times 2)$ periodicity. Apart from a very small difference in the $z$ coordinate of the atoms in sites In 2 and In 3 the top layer is $(4 \times 1)$ reconstructed. This explains why it has been so difficult to see the $c(8 \times 2)$ periodicity in STM images, whereas it was easily visible in diffraction patterns. A second important feature is the two chains in the $[110]_{\text {bulk }}$ direction formed by the atoms on sites In1 and In2,3. They are marked by a red and a green arrow in Fig. 5. In the model of Jones et al. only one of these chains was present. ${ }^{17}$ These authors used a relatively small data set without fractionalorder out-of-plane data and with significantly higher statistical errors (evaluated from the errors given in their Table I). We tried to fit their model to our more complete data set but could not achieve a $\chi^{2}$ below 20; in particular, the fractionalorder rods could not be fitted. The chains in our model are shifted relative to each other by $(0,0.5)_{\text {LEED }}$, i.e., one quarter of the unit cell in the $y$ direction, so they have a different registry with the substrate. The $\mathrm{Sb}$ atoms in the chain marked by the red arrow (sites 7 and 8) are located at bulk positions, 


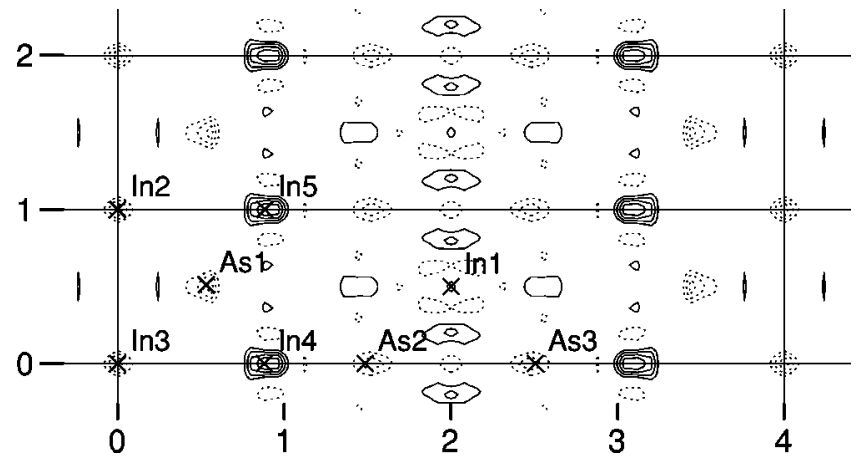

FIG. 8. Difference plot for the InAs data set using a model with only $\mathrm{Nb}$ atoms $(Z=41)$ for the uppermost bilayer. Atomic sites occupied by In atoms $(Z=49)$ appear as positive peaks (solid contour lines), and those occupied by As atoms $(Z=33)$ as negative peaks (dotted contour lines). Crosses mark the positions of the atomic sites.

whereas the indium atoms (site 1) are located on an additional (not bulklike) site. The $\mathrm{Sb}$ atoms in the second chain (site 6, chain marked by a green arrow) are shifted by $(0,0.5)_{\text {LEED }}$ relative to bulk positions, which explains the subsurface dimerization. In a bulk-terminated crystal each In atom in the second bilayer (sites 9 and 10) would have two bonds to $\mathrm{Sb}$ atoms at the surface, as is the case for the atoms on site In10 having bonds to Sb7 and Sb8. Analogously, the In9 atoms would have bonds to two atoms on site Sb6, if they were on bulk sites. But since the entire chain containing the Sb6 atoms is shifted by $(0,0.5)_{\text {LEED }}$ one of the In9-Sb6 bonds is broken, and a dimer bond between neighboring In9 atoms is formed instead. Atomic distances within both chains (In2-Sb6, In3-Sb6, In1-Sb7, and In1-Sb8) are between $3.44 \AA$ and $3.53 \AA$ indicating the metallic character of these bonds (bulk values for In and $\mathrm{Sb}$ are $\sim 3.4 \AA$ ). All other bonds drawn in Fig. 5 represent covalentlike bonds since the corresponding atomic distances ranging from $2.75 \AA$ to $2.88 \AA$ differ from the covalent In-Sb bond length $(2.84 \AA)$ by less than $2.2 \%$.

The solid line in Fig. 6 represents the calculated $(00 l)$ rod based on our model. The agreement with the measured intensities is qualitatively very good: the scattering intensity in the vicinity of the weak (002) bulk reflection is larger than the intensity calculated for a truncated bulk crystal (dashed line) and the interference effect close to the (002) reflection is correctly reproduced. Note that (002) is a very weak bulk reflection, since the scattering from the In and Sb sublattices in the zinc-blende structure almost cancels. Hence the $(00 l)$ rod in the vicinity of (002) almost corresponds to an antiphase condition with high surface sensitivity. The missingdimer model of John et al. ${ }^{6}$ (dotted line in Fig. 6) has less intensity than the truncated crystal. The difference can be explained by the relative In coverage in the top double layer: our model has nominally 16 In atoms, whereas the missingdimer model has a reduced In coverage of only 12 In atoms.

Within the In2-In3 chain an additional site $\operatorname{In} 2 d$ with a reduced occupancy appeared in some of the direct methods maps and could be confirmed by the least-squares refinements. For the InSb data set this site is of minor importance

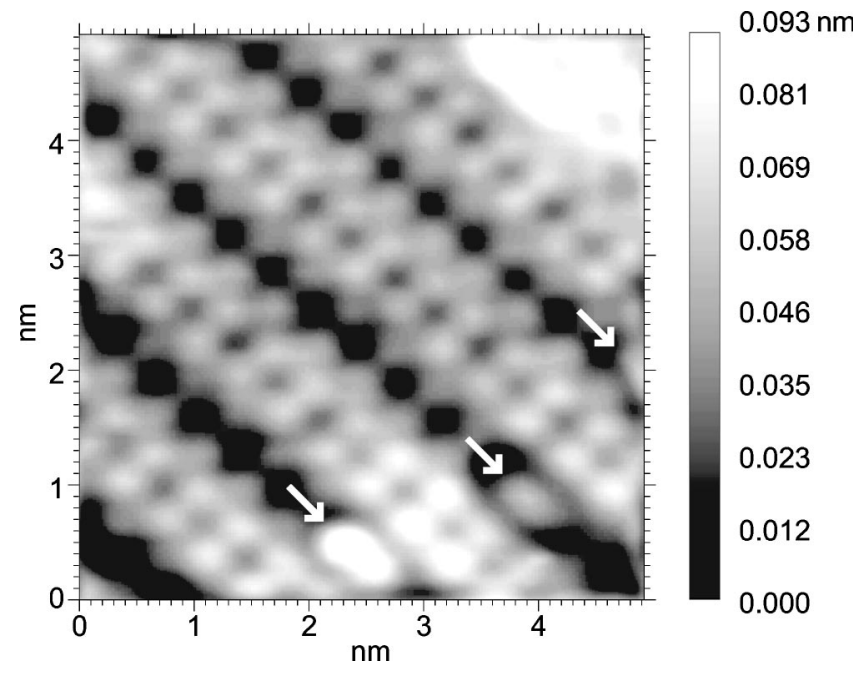

FIG. 9. Filled-state STM image $(-3.0 \mathrm{~V})$ recorded from a $\mathrm{GaAs}(001)$ surface. White arrows mark disorder in the site- 1 chain arising from the reduced occupancy of site 1 .

( $28 \%$ occupancy in contrast to $72 \%$ for the In 2 site and $100 \%$ for In 3 ), whereas in the GaAs structure it is dominant (63\%). The competing sites 2 and 3 are absent in GaAs. This leads to the conclusion that group-III dimerization in the top layer is favored in GaAs, the lighter compound, and reduced in the heavier compounds. Site 1 in the second chain (red arrow) is nearly unoccupied in GaAs (19\%), in contrast to $57 \%$ in InSb. These reduced occupancies are consistent with STM images. Figure 9 shows a filled-state STM image recorded from the GaAs surface with a bias voltage of $-3.0 \mathrm{~V}$. The similarity to the simulated STM image shown by Lee et al. (calculated for $-1.5 \mathrm{~V}$, see the lower left of Fig. 3 in Ref. 18) is striking and indicates that the bright (double) rows correspond to the As atoms on sites 6 in the row marked by the green arrow in Fig. 5, i.e., the rows incompletely occupied by Ga surface dimers on site $2 d$. The dark rows correspond to the rows marked by the red arrow in Fig. 5, where according to our structural refinement a small fraction of the sites 1 is occupied by Ga. However, at some locations marked with white arrows in Fig. 9 bright spots can be seen within the black rows indicating disorder in the site- 1 chain which might be caused by this site being occupied. Although Ga-derived electronic states are probably not imaged at negative sample bias, the contrast visible in Fig. 9 is in agreement with our model of the $\operatorname{GaAs}(001)-c(8 \times 2)$ surface. Reports of disorder caused by partial occupation of some sites exist in the literature, e.g., Fig. 4 of Ref. 14. In the InAs structure the In 1 chain (red arrow in Fig. 5) exhibits a very high degree of disorder, causing diffuse eighth-order reflections.

Our model is consistent with the PES data ${ }^{6}$ which indicated that there is a single In surface electronic state. The structure is also consistent with early ion scattering measurements that show strong evidence of intermixing of group-III and group- $\mathrm{V}$ elements in the surface layer. ${ }^{27}$

\section{SUMMARY}

We have found a unique model for the $c(8 \times 2)$ reconstruction of the group-III-rich (001) surfaces of three differ- 
ent III-V semiconductors (GaAs, InAs, and InSb) using direct methods and subsequent least-squares refinement of SXRD data. It is supported by density-functional-theory calculations, LEED measurements, and simulated STM images. ${ }^{18}$ The reconstruction consists of subsurface dimers of group-III elements in the second bilayer and linear chains of atoms on nonbulklike sites at the surface along the $[110]_{\text {bulk }}$ direction. Within these chains a tendency to dimer formation exists, which decreases with increasing atomic weight of the compounds. We propose that the model describes all metal-rich III-V surfaces.

\section{ACKNOWLEDGMENTS}

We thank the HASYLAB staff for technical assistance. This work was supported by the National Science Foundation with Grant No. DMR09705081 (L.D.M.), the Danish Research Council through Dansync, the German Bundesministerium für Bildung, Forschung und Technologie (BMBF Project No. $05 \mathrm{KS} 1 \mathrm{GUC} / 3$ ), the Deutsche Forschungsgemeinschaft Graduiertenkolleg "Physik nanostrukturierter Festkörper," and the IHP program "Access to Research Infrastructures" of the European Commission (Grant No. HPRI-CT-1999-00040).
*Corresponding author. Present address: Lehrstuhl für Experimentelle Physik II der Universität Würzburg, Am Hubland, D-97074 Würzburg, Germany. FAX: +49 (0)931 888 5158. Email address: kumpf@physik.uni-wuerzburg.de

†Present address: Cornell High Energy Synchrotron Source (CHESS), Wilson Laboratory, Cornell University, Ithaca NY 14853.

${ }^{\ddagger}$ Present address: Saab AB, Future Products \& Technology, Dept. FKC-EL, S-58188 Linköping, Sweden.

${ }^{1}$ C.F. McConville, T.S. Jones, F.M. Leibsle, S.M. Driver, T.C.Q. Noakes, M.O. Schweitzer, and N.V. Richardson, Phys. Rev. B 50, 14965 (1994).

${ }^{2}$ M. Göthelid, Y. Garreau, M. Sauvage-Simkin, R. Pinchaux, A. Cricenti, and G. LeLay, Phys. Rev. B 59, 15285 (1999).

${ }^{3}$ C. Ratsch, W. Barvosa-Carter, F. Grosse, J.H.G. Owen, and J.J. Zinck, Phys. Rev. B 62, R7719 (2000).

${ }^{4}$ V.P. LaBella, H. Yang, D.W. Bullock, P.M. Thibado, P. Kratzer, and M. Scheffler, Phys. Rev. Lett. 83, 2989 (1999).

${ }^{5}$ C. B. Duke, in Physical Structure, Vol. 1 of Handbook of Surface Science, edited by W.N. Unertl (Elsevier North-Holland, Amsterdam, 1996), Chap 6.

${ }^{6}$ P. John, T. Miller, and T.-C. Chiang, Phys. Rev. B 39, 1730 (1989)

${ }^{7}$ J. Cerdá, F.J. Palomares, and F. Soria, Phys. Rev. Lett. 75, 665 (1995).

${ }^{8}$ P.R. Varekamp, M. Bjorkqvist, M. Gothelid, and U.O. Karlsson, Surf. Sci. 350, L221 (1996).

${ }^{9}$ D.K. Biegelsen, R.D. Bringans, J.E. Northrup, and L.-E. Swartz, Phys. Rev. B 41, 5701 (1990).

${ }^{10}$ Q. Xue, T. Hashizume, J.M. Zhou, T. Sakata, T. Ohno, and T. Sakurai, Phys. Rev. Lett. 74, 3177 (1995).

${ }^{11}$ J.E. Northrup and S. Froyen, Phys. Rev. Lett. 71, 2276 (1993).

${ }^{12}$ S. Ohkouchi and N. Ikoma, Jpn. J. Appl. Phys., Part 1 33, 3710 (1994).

${ }^{13}$ C. Kendrick, G. Le Lay, and A. Kahn, Phys. Rev. B 54, 17877 (1996).

${ }^{14}$ M.O. Schweitzer, F.M. Leibsle, T.S. Jones, C.F. McConville, and N.V. Richardson, Surf. Sci. 280, 63 (1993).

${ }^{15}$ A.A. Davis, R.G. Jones, G. Falkenberg, L. Seehofer, R.L. Johnson, and C.F. McConville, Appl. Phys. Lett. 75, 1938 (1999).

${ }^{16}$ S.L. Skala, J.S. Hubacek, J.R. Tucker, J.W. Lyding, S.T. Chou, and K.-Y. Cheng, Phys. Rev. B 48, 9138 (1993).

${ }^{17}$ N. Jones, C. Norris, C.L. Nicklin, P. Steadman, S.H. Baker, A.D. Johnson, and S.L. Bennett, Surf. Sci. 409, 27 (1998).

${ }^{18}$ S.-H. Lee, W. Moritz, and M. Scheffler, Phys. Rev. Lett. 85, 3890 (2000).

${ }^{19}$ C. Kumpf, L.D. Marks, D. Ellis, D. Smilgies, E. Landemark, M. Nielsen, R. Feidenhans'1, J. Zegenhagen, O. Bunk, J.H. Zeysing, Y. Su, and R.L. Johnson, Phys. Rev. Lett. 86, 3586 (2001).

${ }^{20}$ For example, G. Giacovazzo, Direct Methods in Crystallography (Plenum Press, New York, 1980); G. Bricogne, Acta Crystallogr., Sect. A: Found. Crystallogr. 40, 410 (1984); M.M. Woolfson, ibid. 43, 593 (1987); G.M. Sheldrick, ibid. 46, 467 (1990); M.M. Woolfson and H. Fan, Physical and Nonphysical Methods of Solving Crystal Structures (Cambridge University Press, Cambridge, 1995); C.J. Gilmore, Acta Crystallogr., Sect. A: Found. Crystallogr. 52, 561 (1996).

${ }^{21}$ C.J. Gilmore, L.D. Marks, D. Grozea, C. Collazo, E. Landree, and R.D. Twesten, Surf. Sci. 381, 77 (1997); C. Collazo-Davila, L.D. Marks, K. Nishii, and Y. Tanishiro, Surf. Rev. Lett. 4, 65 (1997); E. Landree, C. Collazo-Davila, and L.D. Marks, Acta Crystallogr., Sect. B: Struct. Sci. 53, 916 (1997); L.D. Marks and E. Landree, Acta Crystallogr., Sect. A: Found. Crystallogr. 54, 296 (1998); C. Collazo-Davila, D. Grozea, and L.D. Marks, Phys. Rev. Lett. 80, 1678 (1998); X. Torrelles, J. Rius, F. Boscherini, S. Heun, B.H. Mueller, S. Ferrer, J. Alvarez, and C. Miravitlles, Phys. Rev. B 57, R4281 (1998).

${ }^{22}$ For example, L.G. Gubin, B.T. Polyak, and E.V. Raik, USSR Comput. Math. Math. Phys. 7, 1 (1967); D.C. Youla, in Image Recovery, Theory and Application, edited by H. Stark (Academic Press, Orlando, 1987); M.H. Hayes, ibid.; A. Levi and H. Stark, ibid.; P.L. Combettes, Adv. Imaging Electron Phys. 95, 155 (1996).

${ }^{23}$ L.D. Marks, W. Sinkler, and E. Landree, Acta Crystallogr., Sect. A: Found. Crystallogr. 55, 601 (1999).

${ }^{24}$ L.D. Marks, Phys. Rev. B 60, 2771 (1999).

${ }^{25}$ U. Resch-Esser, N. Esser, D.T. Wang, M. Kuball, J. Zegenhagen, B.O. Fimland, and W. Richter, Surf. Sci. 352-354, 71 (1996).

${ }^{26}$ R. Feidenhans'1, Surf. Sci. Rep. 10, 105 (1989); E. Vlieg, J. Appl. Crystallogr. 30, 532 (1997).

${ }^{27}$ J. Falta, R.M. Tromp, M. Copel, G.D. Pettit, and P.D. Kirchner, Phys. Rev. Lett. 69, 3068 (1992). 